\section{Adult-onset combined methylmalonic aciduria and homocystinuria (cblC)}

O.A.F. Bodamer, MD; D.S. Rosenblatt, MD; S.H. Appel, MD; and A.L. Beaudet, MD

A 20-year-old man was admitted to our service with bilateral paraplegia of the legs, loss of bowel and bladder function, and chronic progressive encephalopathy. He had been well until a year prior to admission when he developed a slowly progressive leg weakness with loss of bowel and bladder control. Subsequently he developed episodes of forgetfulness, sleepiness, and markedly slowed speech. Following several admissions to outlying hospitals the diagnosis of demyelinating disease was suggested and he was treated with corticosteroids. Despite this therapy, his condition deteriorated and he became wheelchair dependent. A month before admission he developed a deep venous thrombosis of his left leg and pelvic veins that required hospitalization and treatment with Coumadin. There was no history of infections or intoxications that might have explained the clinical picture. His medical history was otherwise unremarkable. $\mathrm{He}$ is the only child of nonconsanguineous parents. The family history was noncontributory.

On admission his bulbar musculature strength was normal, but he had weakness and hyperreflexia in the upper extremities and complete paralysis and absent reflexes in the legs. He was considered to have a T8 sensory level to light touch and pinprick. EMG demonstrated scattered denervation in both legs and distal denervation in the left arm and mild chronic denervation of the tongue, all of which were consistent with a diagnosis of motor neuron disease. Eye examination was unremarkable. EEG and brain MRI were normal. CSF studies were unremarkable. There was no anemia or leukocytosis. Plasma vitamin $\mathrm{B}_{12}$ (cobalamin) level was normal. Several days after admission he developed progressive respiratory insufficiency requiring mechanical ventilation. He became lethargic and intermittently unresponsive. The patient also developed occlusion of the femoral and popliteal veins and was assessed for thrombotic disease, which revealed a markedly elevated plasma homocysteine (27.9; normal $5.4-16.2 \mu \mathrm{mol} /$ L). A diagnosis of cobalamin defect was suggested. Urinary organic acid analysis revealed the presence of a very large amount of methylmalonic acid (1722; normal $<2 \mathrm{mmol} / \mathrm{mol}$ creatinine) and the presence of 3-hydroxypropionic acid and methylcitric acid. Complementation analysis of fibroblasts showed that our patient fell into complementation group cblC. Treatment with intramuscular hydroxycobalamin injections and oral carnitine supplementation was started immediately. A low protein diet was commenced. He was weaned from the ventilator within 24 hours of the first hydroxycobalamin dose and had full recovery of mental and upper extremity function within 2 weeks. Urinary methylmalonic acid fell to $12 \mathrm{mmol} / \mathrm{mol}$ creatinine and plasma homocysteine normalized to $15.3 \mu \mathrm{mol} / \mathrm{L}$. However, he was left with residual diplegia and neurogenic bladder and bowel difficulties after 5 years of follow-up. He continues to take $80 \mathrm{mg} / \mathrm{kg} /$ day of carnitine and to have daily IM hydroxycobalamin injections ( $2 \mathrm{mg} /$ day). His protein intake has been normalized.

Combined homocystinuria and methylmalonic aciduria (cblC) is a rare disorder that is due to an unknown defect at an early step of intracellular cobalamin metabolism. Affected children typically present during the neonatal period or early infancy with a history of poor feeding, failure to thrive, recurrent infections, and lethargy. ${ }^{1}$ The mainstay of treatment is intramuscular hydroxycobalamin injections, which are very effective in reducing plasma homocysteine and methylmalonic acid levels but developmental progress may be slow. ${ }^{1}$ Late-onset presentation during adolescence has been reported but, to our knowledge, this is the first case with documented cblC who presented during adulthood. ${ }^{2}$ It can be speculated whether he has a mild mutation in the $c b l C$ gene resulting in significant residual enzyme activity.

The diagnosis of cblC should be considered in any adult presenting with any combination of encephalopathy, myelopathy, motor neuron disease, and thromboembolic events, and consequently plasma homocysteine and methylmalonic acid levels should be measured. Treatment with intramuscular hydroxycobalamin injections and oral carnitine supplementation must be swift to prevent complications and long-term sequelae.

From the Departments of Molecular and Human Genetics (Drs. Bodamer and Beaudet) and Neurology (Dr. Appel), Baylor College of Medicine, Hous ton, TX; and Departments of Human Genetics, Medicine, and Pediatrics (Dr. Rosenblatt), McGill University, Montreal, Canada.

Received October 30, 2000. Accepted in final form January 16, 2001.

Address correspondence and reprint requests to Dr. O.A.F. Bodamer, Assistant Professor, Department of Molecular and Human Genetics, Baylor College of Medicine, Houston, TX 77030; e-mail: obodamer@bcm.tmc.edu

\section{Copyright @ 2001 by AAN Enterprises, Inc.}

\section{References}

1. Rosenblatt DS, Aspler AL, Shevell MI, Pletcher BA, Fenton WA, Seashore MR. Clinical heterogeneity and prognosis in combined methylmalonic aciduria and homocystinuria (cblC). J Inherit Metab Dis 1997;20: $528-538$.

2. Shinnar S, Singer HS. Cobalamin C mutation (methylmalonic aciduria and homocystinuria) in adolescence. A treatable cause of dementia and myelopathy. N Engl J Med 1984;311:451-454.

\section{Cerebral sinus thrombosis with tamoxifen}

\section{Pasquale F. Finelli, MD; and Peter K. Schauer, MD}

The antiestrogen tamoxifen is the most widely used hormonal therapy in the treatment and prophylaxis of breast cancer. ${ }^{1}$ Nervous system complications are rare, ${ }^{2,3}$; however, systemic thrombotic side effects are well described and include deep vein thrombosis and pulmonary embolism. ${ }^{4}$ We report a patient receiving tamoxifen prophylaxis for breast cancer who developed hemorrhagic cerebral infarction secondary to cerebral sinus thrombosis in the absence of other risk factors.

Case report. A 52-year-old woman sought treatment in March 2000 after a 4-day history of progressively worsening right-sided headache. When she became confused, her husband brought her to the Hartford Hospital emergency room, where a CT scan showed a large right temporoparietal hemorrhage. MRI and MR venography showed parenchymal hemorrhage and right transverse sinus thrombosis (figure). Serial MRI over 6 months showed resolution of the parenchymal lesion with persistent sinus thrombosis. Neurologic examination 1 month after her intracerebral hemorrhage showed an unremarkable bedside mental status examination, absent deep tendon reflexes, and marked reduction in vibratory sensation in the lower extremities. Laboratory tests included normal levels of anti-thrombin III activity, protein $\mathrm{C}$ and $\mathrm{S}$ antigen, plasma homocysteine, antiphospholipid antibody panel, activated
Figure. Axial MR fluid-attenuated inversion recovery sequence shows right temporoparietal hemorrhage (A) and increased signal in right sigmoid sinus (arrow) (B). MR venography shows absence of flow in right transverse sinus on time-of-flight sequence (arrows) (C).

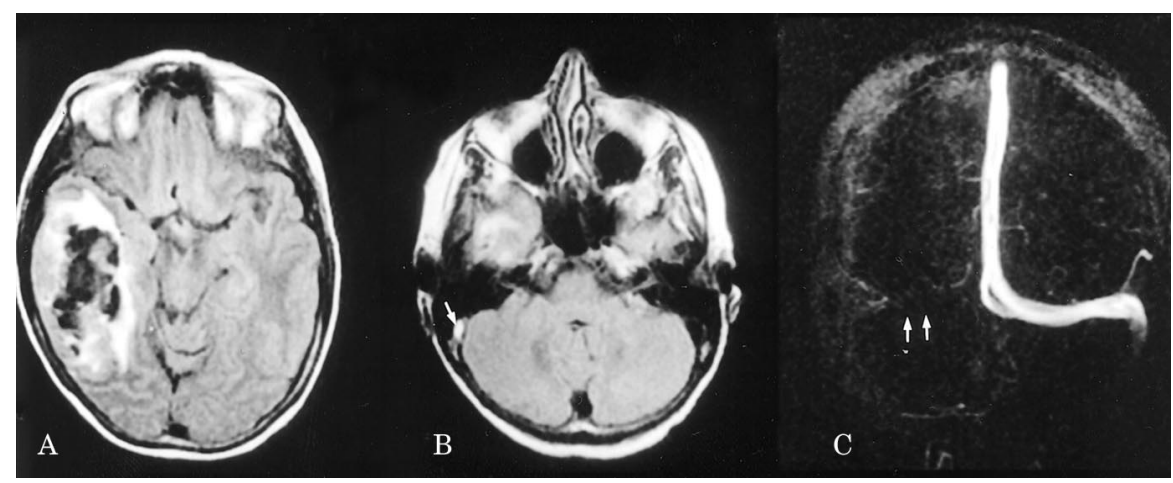

April (2 of 2) 2001 NEUROLOGY $56 \quad 1113$ 